\title{
Linear scleroderma following an intralesional steroid: Was it coincidental?
}

\section{Romita Bachaspatimayum, Singh Shailendra Vikram}

\author{
${ }^{1}$ Department of Dermatology, Venereology and Leprosy, RIMS, Imphal, Manipur, India, ${ }^{2}$ Department of Pharmacology, \\ RIMS, Imphal, Manipur, India
}

Corresponding author: Dr. Romita Bachaspatimayum, E-mail: dr.romita.bachaspatimayum@gmail.com

\begin{abstract}
A 23-year-old female presented herself with a gradual hardening of the skin over the left arm and forearm present for eight months prior. The hardening appeared one month following an intralesional steroid in the left epicondyle area administered for pain and swelling at the site, which were present for three months and associated with painful swelling over the left hand. An examination revealed a depigmented patch over the left lateral epicondyle along with shiny, yellowish, indurated, and hypopigmented to skin-colored plaque extending from the midarm to the wrist over the posterolateral aspect of the upper left extremity. A histopathological examination revealed features suggestive of scleroderma. The patient was diagnosed with linear scleroderma and treated with methotrexate and hydroxychloroquine with significant improvement after six weeks.
\end{abstract}

Key words: Steroid; Scleroderma; Hypopigmentation

\section{INTRODUCTION}

Corticosteroids are the mainstay of the treatment of scleroderma. Out of the many side effects of intralesional steroids-including hypopigmentation, skin atrophy, hypertrichosis, and telangiectasia—skin sclerosis and scleroderma have not yet been reported. Herein, we report a case of linear scleroderma, which appeared one month following an intralesional injection of a corticosteroid. Whether it was because of the steroid itself or because of its indirect inducement remains unknown.

\section{CASE REPORT}

A 23-year-old female presented herself with a gradual hardening of the skin over the left arm and forearm present for eight months prior. The hardening appeared one month following an intralesional steroid-40 mg triamcinolone acetonide-in the left epicondyle area administered for pain and swelling at the site, which had been present for three months since the injection.
This was associated with a painful swelling of the left hand along with the weakness of the left hand, mainly the left ring finger. There was no history of Raynaud's phenomenon or other systemic symptoms. An examination revealed a depigmented patch $3 \times 2.5 \mathrm{~cm}$ in size over the left lateral epicondyle, adjacent to several scaly papules coalescing into a plaque, along with a shiny, yellowish, indurated, and hypopigmented to skin-colored plaque extending from the midarm to the wrist over the posterolateral aspect of the upper left extremity. A tender swelling $5 \times 7.5$ $\mathrm{cm}$ in size was present over the dorsum of the left hand with normal overlying skin but with weakness in the extension of the middle and ring fingers (Figs. 1-3). A systemic examination was normal. A histopathological examination revealed a marked increase in collagen with a paucity of adnexal structures in the dermis and dermal collagen intersecting into the subcutaneous fat, which are features suggestive of scleroderma (Fig. 4). An immunofluorescence assay for antinuclear antibody (ANA) was positive $(1+)$, with cytoplasmic patterns with a titer of 1:80. The level of serum vitamin $D$ was

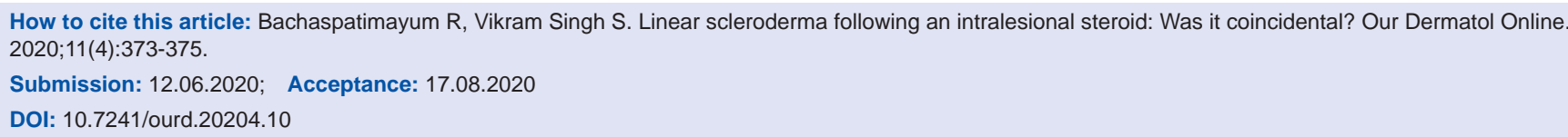




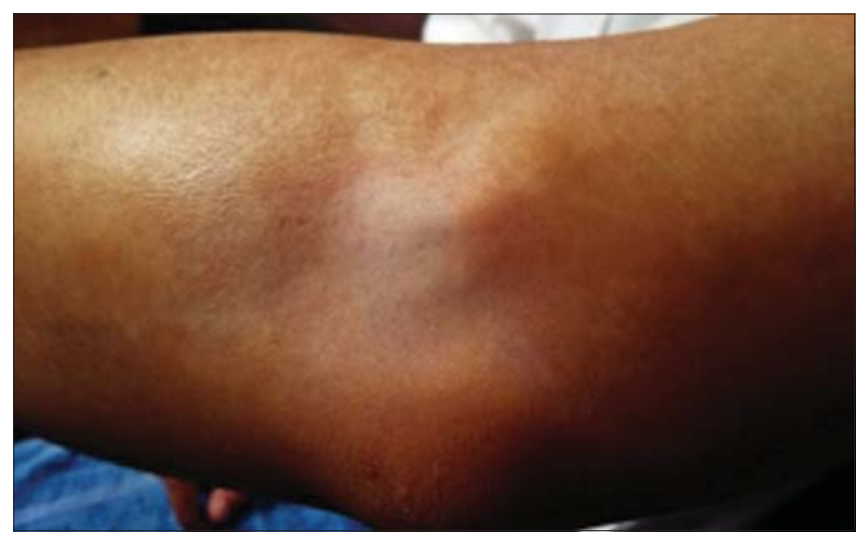

Figure 1: A depigmented irregular patch $3 \times 2.5 \mathrm{~cm}$ in size over the left lateral epicondyle.

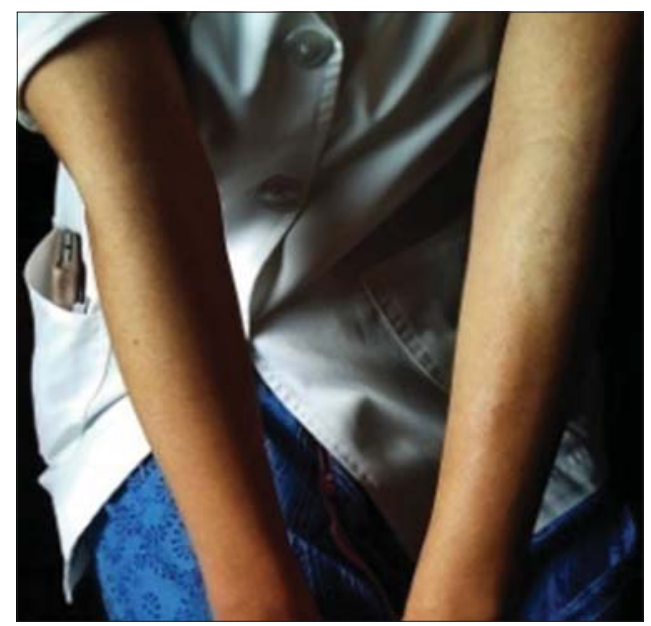

Figure 2: A shiny, yellowish, indurated, and hypopigmented to skin-colored plaque extending from the midarm to the wrist over the posterolateral aspect of the upper left limb.

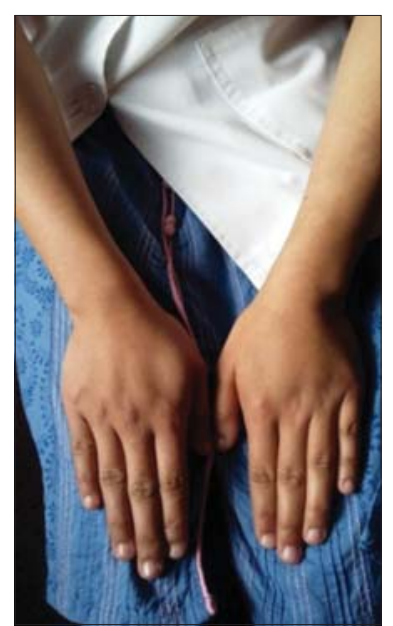

Figure 3: A tender swelling $5 \times 7.5 \mathrm{~cm}$ in size over the dorsum of the left hand with normal overlying skin.

insufficient $(26 \mathrm{ng} / \mathrm{mL})$. Other routine investigationsincluding anti-dsDNA (anti-double stranded DNA) antibodies, anti-centromere antibodies, and an X-ray

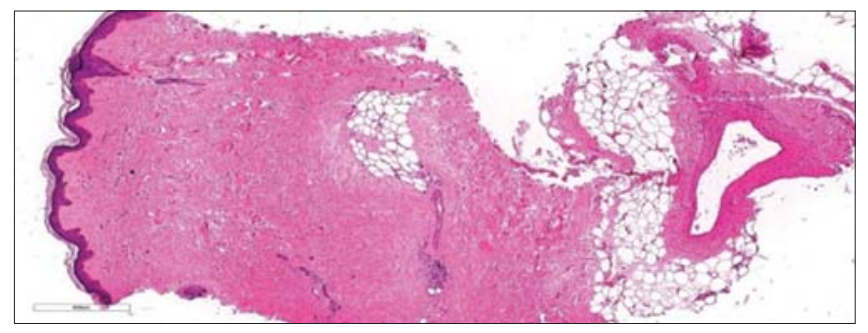

Figure 4: A histopathological examination showing a marked increase in collagen with a paucity of adnexal structures in the dermis and dermal collagen intersecting into the subcutaneous fat, revealing features suggestive of scleroderma (H\&E, 10x).

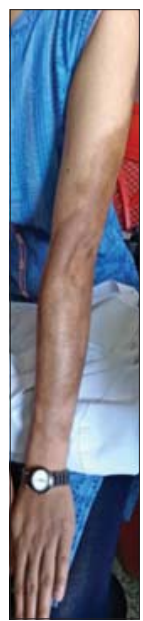

Figure 5: The last follow-up (one year after) with significant softening of the induration and repigmentation of the depigmented areas of the plaque.

of the left hand in an anteroposterior and lateral view-were normal. The patient was diagnosed with linear scleroderma and treated with oral methotrexate $15 \mathrm{mg} /$ week, hydroxychloroquine $200 \mathrm{mg} \mathrm{BD}$, and $0.1 \%$ topical tacrolimus BD with significant improvement by week four (skin softening and repigmentation of the depigmented and hypopigmented areas) with regular monitoring of the hematocrit and liver function. However, the brownish discoloration without skin tightening began to expand over the left shoulder by the second month and stopped after three weeks. The patient is still on regular follow-ups with no significant adverse effects. Fig. 5 shows the clinical photograph after one year of treatment when significant softening of the induration was found along with repigmentation of the depigmented areas of the plaque.

\section{DISCUSSION}

A systematic review of literature by Brinks et al. [1] lists the dermal adverse events of local corticosteroid injections to include irritation, changes 
in skin color, skin and perilymph atrophy, soft tissue calcification, skin defects, hypopigmentation, sterile abscesses, ecchymosis, and allergic rash, and the infectious adverse events to include cellulitis, localized abscesses, septic bursitis, atypical mycobacterium infections, necrotizing fasciitis, and protothecosis. The local adverse events include local pain, tingling or numbness in the hands, local neural damage, and tendon rupture, and the systemic adverse events include allergic reactions, facial flushing, and disturbance of the menstrual pattern. Hypopigmentation and skin atrophy can occur following local steroid injections, although the mechanism by which hypopigmentation occurs is unclear [2]. Physical injury, including local injections, has also been described as a possible provoking factor of localized scleroderma [3]. Skin induration can also occur, besides systemic sclerosis, in localized forms of scleroderma as well as in conditions unrelated to scleroderma or systemic sclerosis, classified as scleroderma variants. These conditions are rarely associated with sclerodactyly or Raynaud's phenomenon, and autoantibody formation does not occur [4]. In our case, the assay for ANA, although positive, was insignificant. Thus, whether the onset of the disease after the steroid injection was coincidental or was provoked by trauma from the injection is yet to be elucidated, although the latter seems likely. In systemic sclerosis, microvascular injury is a very early event and the same changes may occur in morphea [5]. Mechanical injury may have caused vascular endothelial damage and neuronal injury, which could have contributed to the development of trauma-induced scleroderma [3]. In any case, we recommend further research in this pursuit.

\section{CONSENT}

The examination of the patient was conducted according to the principles of the Declaration of Helsinki.

The authors certify that they have obtained all appropriate patient consent forms, in which the patients gave their consent for images and other clinical information to be included in the journal. The patients understand that their names and initials will not be published and due effort will be made to conceal their identity, but that anonymity cannot be guaranteed.

\section{REFERENCES}

1. Brinks A, Koes BW, Volkers ACW, Verhaar JAN, BiermaZeinstra SMA. Adverse effects of extra-articular corticosteroid injections: a systematic review. BMC Musculoskelet Disord. 2010;206:1-11.

2. Coondoo A, Phiske M, Verma S, Lahiri K. Side-effects of topical steroids: a long overdue revisit. Indian Dermatol Online J. 2014;5:416-25.

3. Komócsi A, Tóvári E, Kovács J, Czirják L. Physical injury as a provoking factor in three patients with scleroderma. Clin Exp Rheumatol. 2000;18:622-4.

4. Simms WR. Localized scleroderma and scleroderma like syndromes. In: Hochberg MC, Silman AJ, Smolen JS, Weinblatt ME, Weisman $\mathrm{MH}$, editors. Rheumatology $6^{\text {th }} \mathrm{Ed}$. Philadelphia: Elsevier Mosby; 2015. p. 1219-23.

5. Rocken M, Ghoreschi K. Morphea and lichen sclerosus. In: Bolognia JL, Jorizzo JL, Schaffer JV, editors. Dermatology $3^{\text {rd }}$ Ed. New Delhi: Reed Elsevier India Private Limited; 2012. p. 657-70.

Copyright by Romita Bachaspatimayum, et al. This is an open-access article distributed under the terms of the Creative Commons Attribution License, which permits unrestricted use, distribution, and reproduction in any medium, provided the original author and source are credited.

Source of Support: Nil, Conflict of Interest: None declared. 